\title{
THE ODLYZKO CONJECTURE AND O'HARA'S UNIMODALITY PROOF
}

\author{
DENNIS STANTON AND DORON ZEILBERGER
}

(Communicated by Andrew M. Odlyzko)

\begin{abstract}
We observe that Andrew Odlyzko's conjecture that the Maclaurin coefficients of $1 /\left[(1+q)\left(1+q+q^{2}\right) \cdots\left(1+q+\cdots+q^{k-1}\right)\right]$ have alternating signs is an almost immediate consequence of an identity that is implied by Kathy O'Hara's recent magnificent combinatorial proof of the unimodality of the Gaussian coefficients.
\end{abstract}

To a true combinatorialist, a combinatorial result is not properly proved until it receives a direct combinatorial proof. This is why Kathy O'Hara's long-soughtfor constructive proof of the unimodality of the Gaussian polynomials ([4], [5], see also [6]) generated so much excitement in combinatorial circles. However to non-combinatorialists, a direct combinatorial proof is "just another proof." O'Hara's proof is longer than most of the dozen previous proofs, and probably would not add any insight to anyone who is not a genuine combinatorialist. Moreover, it does not seem to be generalizable at first sight. Yet it turned out to imply a deep result $(\mathrm{KOH})$ to which hitherto there was no known proof of any kind.

In this note we shall prove and generalize a conjecture of Odlyzko, using O'Hara's result. Odlyzko's results imply that for $k$ sufficiently large, the first $k$ coefficients in

$$
\frac{1}{(1+q)\left(1+q+q^{2}\right) \cdots\left(1+q+\cdots+q^{k-1}\right)}=\frac{(1-q)^{k}}{(1-q)\left(1-q^{2}\right) \cdots\left(1-q^{k}\right)}
$$

alternate in sign. He conjectured that in fact for every $k \geq 0$, all of the coefficients of the above series alternate in sign. We prove the sharper result

Theorem 1. For any integer $k$,

$$
\frac{(1-q)^{[(k+1) / 2]}}{(1-q)\left(1-q^{2}\right) \cdots\left(1-q^{k}\right)}
$$

has coefficients which alternate in sign.

Received by the editors March 28, 1988 and, in revised form, November 28, 1988.

1980 Mathematics Subject Classification (1985 Revision). Primary 05A17, 05A20, 05A30, 10 A45.

The first author's work was partially supported by NSF grant DMS:8700995.

The second author's work was partially supported by NSF grant DMS: 8800663 . 
Note that the exponent of $(1-q)$ is best possible, since if $[(k+1) / 2]$ is replaced by $[(k-1) / 2]$ then the pole $q=1$ has the highest order among all the poles, all of which are roots of unity, so a partial fraction expansion would yield that the coefficients are asymptotically of the same sign.

Odlyzko has informed the authors that Theorem 1 can be used to shorten the proof in [3] by at least one third.

We will prove a more general result. Recall that the Gaussian polynomials are defined for nonnegative integers $k$ and $n$ by

$$
G(n, k)=\left[\begin{array}{c}
n+k \\
k
\end{array}\right]_{q}=\frac{\left(1-q^{n+1}\right)\left(1-q^{n+2}\right) \cdots\left(1-q^{n+k}\right)}{(1-q)\left(1-q^{2}\right) \cdots\left(1-q^{k}\right)} .
$$

If $n$ is negative, we put $G(n, k)=0$. We will prove:

Theorem 2. For nonnegative integers $n$ and $k$, with $n k$ even, $G(n, k)(1-q)^{m}$ has coefficients which alternate in sign, where $m=\min \{[(k+1) / 2],[(n+1) / 2]\}$.

Theorem 1 follows from Theorem 2 upon taking $n$ even and letting $n \rightarrow \infty$.

Theorem 2 will follow from the following amazing $q$-binomial identity that was derived in [7], by "algebrizing" O'Hara's main theorem ([4], [5], [6]).

$$
G(n, k)=\sum_{\lambda \vdash k} q^{2 n(\lambda)} \prod_{i=0}^{k-1} G\left((k-i) n-2 i+\sum_{j=0}^{i-1} 2(i-j) d_{k-j}, d_{k-i}\right),
$$

where

$$
n(\lambda)=\sum_{i}(i-1) \lambda_{i}
$$

The sum in $(\mathrm{KOH})$ is over all partitions $\lambda=\left(\lambda_{1}, \lambda_{2}, \ldots\right)$ of $k$. The integer $d_{i}$ is the multiplicity of $i$ in $\lambda$, thus in frequency notation $\lambda=1^{d_{1}} 2^{d_{2}} \cdots i^{d_{i}} \cdots$. In this notation,

$$
2 n(\lambda)=\sum_{i=1}^{k}\left(D_{i}^{2}-D_{i}\right)
$$

where

$$
D_{r}=\sum_{i=r}^{k} d_{i}
$$

Proof of Theorem 2. By symmetry in $n$ and $k$, we may assume that $n$ is even. We proceed by induction on $n$ and $k$. Theorem 2 clearly holds for $n=0$ and $k=1$.

Let

$$
F(n, k):=(1-q)^{[(k+1) / 2]} G(n, k) .
$$

Then $(\mathrm{KOH})$ can be rewritten as $\left(\mathrm{KOH}^{\prime}\right)$

$$
F(2 n, k)=\sum_{\lambda \vdash k}(1-q)^{\alpha(\lambda)} q^{2 n(\lambda)} \prod_{i=0}^{k-1} F\left(2(k-i) n-2 i+\sum_{j=0}^{i-1} 2(i-j) d_{k-j}, d_{k-i}\right) .
$$


where

$$
\alpha(\lambda):=m-\sum_{i=1}^{k}\left[\left(d_{i}+1\right) / 2\right] .
$$

Suppose we show that $\alpha(\lambda) \geq 0$. If $d \neq 1^{k}$, then each $F$ on the right side of $\left(\mathrm{KOH}^{\prime}\right)$ has a second argument less than $k$. If $\lambda=1^{k}$, the first argument of $F$ is less than $2 n$. Thus by induction each $F$ is alternating. Since $(1-q)^{\alpha(\lambda)}$ is alternating, and the power of $q$ is even, the left side must be alternating. So it remains to verify that $\alpha(\lambda) \geq 0$.

First suppose that $n \geq[(k+1) / 2]$, so $m=[(k+1) / 2]$. Then we will show that for any partition $\lambda$ of $k$, we have the inequality

$$
[(k+1) / 2]-\sum_{i=1}^{k}\left[\left(d_{i}+1\right) / 2\right] \geq 0 .
$$

It is easy to see that $(*)$ is

$$
[(k+1) / 2]-\left(\text { number of parts of } \lambda+\text { number of } i \text { with } d_{i} \text { odd }\right) / 2 \text {. }
$$

This is nonnegative, since any part $i>1$ of $\lambda$ can contribute at most one $i$ which has $d_{i}$ odd.

Next suppose that $n<[(k+1) / 2]$, so $m=n$. First we show

$$
n+1-\sum_{i=1}^{k} d_{i} \geq 0
$$

for all partitions $\lambda$ of $k$ which occur in $\left(\mathrm{KOH}^{\prime}\right)$. The key observation is that $F$ is zero if the first argument is negative. Thus, taking the $i=k-1$ term in $\left(\mathrm{KOH}^{\prime}\right)$, we see that

$$
2 n-2(k-1)+\sum_{j=0}^{k-2} 2(k-1-j) d_{k-j} \geq 0,
$$

which is equivalent to

$$
\sum_{j=2}^{k}(j-1) d_{j} \geq k-1-n
$$

or

$$
k=\sum_{j=1}^{k} j d_{j} \geq k-1-n+\text { number of parts of } \lambda .
$$

The final inequality implies that $\lambda$ has at most $n+1$ parts, which is $(* *)$. Clearly $\alpha(\lambda) \geq 0$ holds unless $\lambda$ has $n+1$ distinct parts, in which case $\alpha(\lambda)=$ -1 . In this case the $i=k-1$ term in $\left(\mathrm{KOH}^{\prime}\right)$ is alternating $(G(0,1)=1)$ without the factor of $(1-q)$, so it is enough to prove that $\alpha(\lambda)+1 \geq 0$.

Remarks. To prove Theorem 1 we need only the $n \rightarrow \infty$ case of $(\mathrm{KOH})$. John Stembridge rediscovered an identity of Hall which implies this result:

$$
\left[\begin{array}{c}
n+k \\
k
\end{array}\right]_{q}=\sum_{d \vdash k} q^{2 n(d)}\left[\begin{array}{c}
n+1 \\
d_{1}, \ldots, d_{k}
\end{array}\right]_{q} \text {. }
$$


Then George Andrews observed that (JS) is nothing but an iteration of $q$ Vandermonde. Subsequently John Stembridge and Jim Joichi gave bijections that prove (JS). Their proofs are closely related to [1].

If $n k$ is odd, Theorem 2 cannot hold, because the leading term has the wrong sign. The exponent in Theorem 2 is not always best possible: $G(11,6)(1-q)^{2}$ alternates in sign.

Ron Evans has made the following related conjecture. He has verified it for $a=1$ from Theorem 2 .

Conjecture. Let $n, k$, and a be nonnegative integers, with $k>3$ and a odd. Let $G(n, k, a)$ be defined by $(G P)$, with $q^{a}$ replacing $q$ in the numerator. Then the coefficients of $G(n, k, a)(1-q)^{[(k+1) / 2]}$ alternate in sign if $n k$ is even, and the coefficients of $G(n, k, a)(1-q)^{[(k+1) / 2]} /\left(1-q^{2}\right)$ alternate in sign if $n k$ is odd.

Some other remarks about $(\mathrm{KOH})$ can be found in [7].

\section{REFERENCES}

1. G. Andrews, Partitions and Durfee dissection, Amer. J. Math., 101 (1979), pp. 735-742.

2. I. G. Macdonald, Symmetric functions and Hall polynomials, Clarendon Press, Oxford, 1979.

3. A. Odlyzko, On differences of the partition function, Acta Arith., 49 (1988), pp. 237-254.

4. K. M. O'Hara, Unimodality of Gaussian coefficients: a constructive proof, research announcement.

5. __ Unimodality of Gaussian coefficients: a constructive proof, J. Combin. Theory Ser. A (to appear).

6. D. Zeilberger, Kathy O'Hara's constructive proof of the unimodality of the Gaussian polynomials, Amer. Math. Monthly (to appear).

7. $\ldots$, A one-line high school algebra proof of the unimodality of the Gaussian polynomials $\left[\begin{array}{l}n \\ k\end{array}\right]_{q}$ for $k<20$, Proceedings of the IMA Workshop on $q$-series, Vol. 18, Springer.

School of Mathematics, University of Minnesota, Minneapolis, Minnesota 55455

Department of Mathematics, Drexel University, Philadelphia, Pennsylvania 19104 